\title{
Denoising Method Based on the Nonsubsampled Shearlet Transform
}

\author{
Xiaoxia Ren ${ }^{1, a}$, Zuoyu Wei ${ }^{1, a}$, Zhifang He ${ }^{2, b}$, Xiuming Sun ${ }^{1, a}$, Peng Geng ${ }^{3, c}$ \\ ${ }^{1}$ Science Department, Zhanjiakou University, Zhanjiakou, 075000, China \\ ${ }^{2}$ Computer Department, TangShan vocational College of Science and Technology, Tangshan, \\ 063001, China \\ ${ }^{3}$ School of information sciences and technology, Shijiazhuang Tiedao University, Shijiazhuang, \\ 050000, China \\ aemail: 171314720@qq.com ${ }^{\text {bemail: xiaowntong@163.com }{ }^{c} e m a i l: 28131230 @ 163 . c o m ~}$
}

Keywords: nonsubsampled Shearlet; Bivariate Shrinkage; image denoising

\begin{abstract}
In this paper, a new bivariate shrinkage denoising method is proposed to model statistics of shearlet coefficients of images. Using Bayesian estimation theory we derive from this model a simple non-linear shrinkage function for shearlet denoising, which generalizes the soft threshold approach. Experimental results show that the proposed method can remove Gaussian white noise while effectively preserving edges and texture information. At the same time, it can achieve a higher PSNR and mean structural similarity than other denoising method.
\end{abstract}

\section{Introduction}

Nowadays, lots of research work on image processing is concentrated in the multiscale transform. Wavelet threshold has been presented as a true signal estimation technique that exploits the capabilities of wavelet transform (DWT) for signal denoising [1]. After this, a theory for multidimensional data called as MGA has been developed such as curvelet, bandelet, contourlet, Nonsubsampled Contourlet Transform (NSCT) [1] etc.. These new MGA tools provide higher directional sensitivity than wavelets. However, the curvelet, bandelet, contourlet lack of shift-invariance and results in artifacts along the edges to some extend. To represent the edges more efficiently, Labate et al. introduced a new multiscale analysis tool called shearlet that has all properties like other MGA tools as multiscale, localization, anisotropy and directionality [2]. The nonsubsampled shearlet transform (NSST) is realized by nonsubsampled Laplacian pyramid (NSLP) and several shearing filters. The NSST also provides the flexible directional selectivity and shift invariance.

\section{Bivariate Shrinkage}

Let $\omega_{2}$ be parent shearlet coefficient and $\omega_{1}$ be child coefficient [3].

$$
\begin{gathered}
y_{1}=\omega_{1}+\varepsilon_{1} \\
y_{2}=\omega_{2}+\varepsilon_{2}
\end{gathered}
$$

Where $\varepsilon_{1}, \varepsilon_{2}$ are the independent Gaussian noise coefficient. $y_{1}, y_{2}$ are observations of the shearlet coefficient of noisy image, respectively. $\omega_{1}, \omega_{2}$ represent the shearlet coefficient of the ideal clean image. Then, the shearlet coefficient can be expressed as $y=\omega+\varepsilon$. Where $\varepsilon=\left(\varepsilon_{1}, \varepsilon_{2}\right), \quad y=\left(y_{1}, \mathrm{y}_{2}\right), \quad \omega=\left(\omega_{1}, \omega_{2}\right)$.The standard maximum a posterior estimator for $\omega$, given the noisy observation is $\hat{\omega}(y)=\arg \max P_{\omega \mid y}(\omega \mid y)$. The noisy observation express as $\widehat{\omega}(y)=\arg \max \left[\log p_{\varepsilon}(y-\omega)+\log \left(p_{\varepsilon}(\omega)\right)\right]$. Noise $p_{\varepsilon}$ is assumed as identically Gaussian independent distributed with 0 mean and $\sigma_{\varepsilon}^{2}$ variance. The noise pdf can be written as 
$p_{\varepsilon}=\frac{1}{2 \pi \sigma_{\varepsilon}^{2}} \exp \left(-\frac{\varepsilon_{1}^{2}+\varepsilon_{2}^{2}}{2 \sigma_{\varepsilon}^{2}}\right)$. In order to observe, pdf of shearlet coefficients $p_{\omega}(\omega)$, we use the joint empirical child-parent coefficients. we propose the following pdf: $p_{\omega}(\omega)=\frac{3}{2 \pi \sigma^{2}} \exp \left(-\frac{\sqrt{3}}{\sigma} \sqrt{\omega_{1}^{2}+\omega_{2}^{2}}\right)$.

Let $f(\varepsilon)=\log \left(p_{\varepsilon}(\varepsilon)\right) . \hat{\omega}(y)=\arg \max \left[-\frac{\left(y_{1}-\omega_{1}\right)^{2}}{2 \sigma_{\varepsilon}^{2}}-\frac{\left(y_{2}-\omega_{2}\right)^{2}}{2 \sigma_{\varepsilon}^{2}}+f(\omega)\right]$ can be calculated. If $p(\varepsilon)$ is assumed to be strictly convex and differentiable, the solving process can be converted into the equations:

$$
\begin{aligned}
& \frac{y_{1}-\widehat{\omega}_{1}}{\sigma_{\varepsilon}^{2}}+f_{1}(\widehat{\omega})=0 \\
& \frac{y_{2}-\widehat{\omega}_{2}}{\sigma_{\varepsilon}^{2}}+f_{2}(\widehat{\omega})=0
\end{aligned}
$$

Where $y_{1}, y_{2}$ denote for the partial derivatives $f(\omega)$. If $f(\omega)=\log \left(\frac{3}{2 \sigma^{2}}\right)-\frac{3}{\sigma} \sqrt{\omega_{1}^{2}+\omega_{2}^{2}}$ is substituted into Eq. (3) and Eq. (4), the MAP estimator or the joint shrinkage function can be writing as,

$$
\widehat{\omega}_{1}=\frac{\left(\sqrt{y_{1}^{2}+y_{2}^{2}}-\frac{\sqrt{3} \sigma_{\varepsilon}^{2}}{\sigma}\right)}{\sqrt{y_{1}^{2}+y_{2}^{2}}} \square y_{1}
$$

\section{Proposed method}

The NSST is an extension of the Wavelet transform in multidimensional and multidirectional case which combines the multiscale and direction analysis, separately. Firstly, the nonsubsampled Laplacian pyramid (NSLP) is used to decompose an image into low and high-frequency components, and then direction filtering is employed to get the different subbands and different direction shearlet coefficients. Direction filtering is achieved using the shear matrix which provides many more directions. In order to eliminate the effect of up-sampling and sub-sampling, NSST adopted NSLP filters as a substitute for the Laplacian pyramid filters used in the Shearlet transform, so that it has superior performance in shift-invariance, multiscale and multidirectional properties. In this study, the proposed denoising image method combined with bivariate shrinkage is applied on every detailed coefficients of the NSST decomposition. Denoising algorithm is mainly composed of follows steps, namely: Firstly, perform the multiscale decomposition adopting the nonsubsampled shearlet transform to obtain the noisy NSST coefficients. Secondly, Apply the threshold scheme on the noisy nonsubsampled shearlet transform coefficients to get the threshold shearlet. Finally, invert the multiscale decomposition to reconstruct the denoised image.

\section{Experimental results}

To demonstrate the effectiveness of the method further, we compare it with three denoising algorithms including BLS-GSM [5], (SA-DCT) [6] and Bivariate NSCT [7]. Fig. 1 shows that the BSL-GSM performs the worst effect among these denoising methods. After denoising some textures of the edge are removed by the denoising method. And the denoising method in the SA-DCT has a slightly artificial texture. Lastly, the proposed algorithm in this paper has a better effect in maintaining the image edge and texture than these others. What is more, it suppresses the 
generation of artificial texture. In order to objectively evaluate these algorithms, PSNR denoising performance parameters is calculated shown in Table 1. From Table 1, we can know that the proposed method performs better.

Table 1: Denoising results comparison of various methods

$\begin{array}{ccccccc}\text { Image } & \sigma & \text { Noisy } & \text { BSL-GSM } & \begin{array}{c}\text { Bivariate } \\ \text { NSCT }\end{array} & \text { SA-DCT } & \begin{array}{c}\text { Proposed } \\ \text { Method }\end{array} \\ & 20 & 22.1029 & 32.8576 & 32.6952 & 32.8412 & \mathbf{3 3 . 7 6 6 3} \\ \text { Lena } & 30 & 18.5779 & 31.0579 & 30.7844 & 31.0182 & \mathbf{3 1 . 2 5 9 7} \\ & 50 & 14.1424 & 28.7720 & 28.3083 & 28.7819 & \mathbf{2 8 . 6 9 8 1} \\ & 20 & 22.0977 & 30.2607 & 30.5538 & 29.9811 & \mathbf{3 0 . 8 9 6 3} \\ \text { Barbara } & 30 & 18.6013 & 28.1944 & 28.4924 & 28.1321 & \mathbf{2 8 . 6 3 8 4} \\ & 50 & 14.1566 & 25.4616 & 25.7705 & 25.5011 & \mathbf{2 5 . 8 8 1 9}\end{array}$

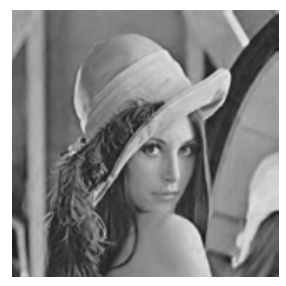

(a) BSL-GSM

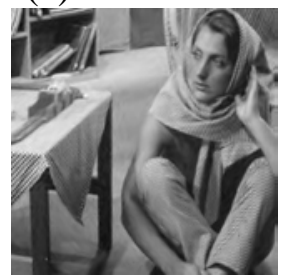

(a) BSL-GSM

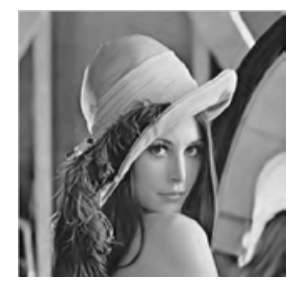

(b) Bivariate NSCT

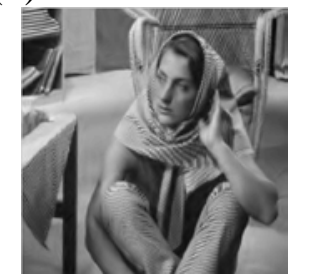

(b) Bivariate NSCT

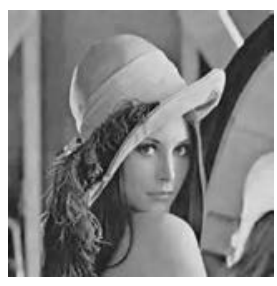

(d) SA-DCT

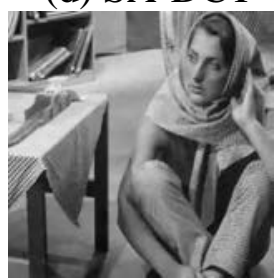

(d) SA-DCT

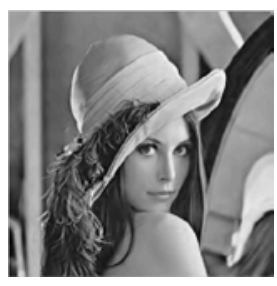

(e) Proposed Method

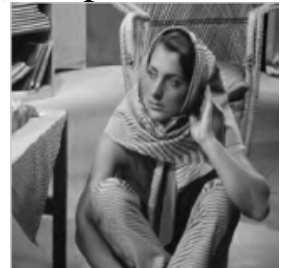

(e) Proposed Method

Fig. 1 Denoised result by several methods

\section{Conclusion}

The coefficient in the multiscale transform domain is directly thresholded in the traditional shrinkage denoising method ignoring the correction between the different scales. According to the characteristic of the nonsubsampled shearlet transform, the coefficient correction between the parent scale and child scale is introduced into the shearlet coefficient. The experiments demonstrate the present method is more effective than the traditional denoising methods are.

\section{References:}

[1]Ming Yin,Wei Liu, Xia Zhao, Yanjun Yin, Yu Guo. A novel image fusion algorithm based on nonsubsampled shearlet transform [J]. Optik - International Journal for Light and Electron Optics. 2014, 125(10): 2274-2282

[2]Geng Peng, Wang Zhengyou, Zhang Zhigang, Xiao Zhong. Image fusion by pulse couple neural network with shearlet. Optical Engineering, v 51, n 6, June 2012

[3]Songfeng Yin, Liangcai Cao, Yongshun Ling. Image denoising with anisotropic bivariate shrinkage [J]. Signal Processing. 2011, 91(8): 2078-90

[4] Pan Jinfeng, Pan Xuefeng. The Adaptive Bivariate Shrinkage Denoising Method [C]. International Conference on Artificial Intelligence and Computational Intelligence, Sanya, 2010, 23-24 
[5]Portilla Javier, Strela Vasily, Wainwright Martin J., Simoncelli Eero P. Image denoising using scale mixtures of Gaussians in the wavelet domain[J]. IEEE Transactions on Image Processing, 2003, 12(11): 1338-1351

[6]Foi Alessandro, Katkovnik Vladimir, Egiazarian Karen. Pointwise shape-adaptive DCT for high-quality denoising and deblocking of grayscale and color images[J]. IEEE Transactions on Image Processing, 2007, 16(5): 1395-1411

[7]Jia Jian, Jiao LiCheng, Xiang, HaiLin. Using bivariate threshold function for image denoising in NSCT domain [J]. Journal of Electronics and Information Technology, 2009 ,31(3): 532-536 